\title{
Single-Band Model of Resonant Inelastic X-Ray Scattering by Quasiparticles in High-Tc Cuprate Superconductors
}

\section{Citation}

Benjamin, David, Israel Klich, and Eugene Demler. 2014. "Single-Band Model of Resonant Inelastic X-Ray Scattering by Quasiparticles in High-Tc Cuprate Superconductors." Physical Review Letters 112 (24) (June). doi:10.1103/physrevlett.112.247002.

\section{Published Version}

10.1103/PhysRevLett.112.247002

\section{Permanent link}

http://nrs.harvard.edu/urn-3:HUL.InstRepos:22856732

\section{Terms of Use}

This article was downloaded from Harvard University's DASH repository, and is made available under the terms and conditions applicable to Other Posted Material, as set forth at http:// nrs.harvard.edu/urn-3:HUL.InstRepos:dash.current.terms-of-use\#LAA

\section{Share Your Story}

The Harvard community has made this article openly available.

Please share how this access benefits you. Submit a story.

\section{Accessibility}




\title{
Single-Band Model of Resonant Inelastic X-Ray Scattering by Quasiparticles in High- $T_{c}$ Cuprate Superconductors
}

\author{
David Benjamin, ${ }^{1}$ Israel Klich, ${ }^{2}$ and Eugene Demler ${ }^{1}$ \\ ${ }^{1}$ Physics Department, Harvard University, Cambridge, Massachusetts 02138, USA \\ ${ }^{2}$ Department of Physics, University of Virginia, Charlottesville, Virginia 22904, USA
}

(Received 23 December 2013; published 18 June 2014)

\begin{abstract}
We show that a simple model of noninteracting quasiparticles accurately describes resonant inelastic $\mathrm{x}$-ray scattering (RIXS) experiments in the hole-doped cuprate superconductors. Band structure alone yields signatures previously attributed to collective magnetic modes, such as the dispersing peaks and nontrivial polarization dependence found in several experiments. We conclude that RIXS data can be explained without positing the existence of magnetic excitations that persist with increasing doping. In so doing we develop a formalism for RIXS in itinerant electron systems that accounts for the positively charged core hole exactly and discover a mechanism by which the core hole produces polarization dependence mimicking that of a magnetic system.
\end{abstract}

DOI: 10.1103/PhysRevLett.112.247002

PACS numbers: 74.72.Gh, 78.70.Ck

It is widely believed that collective magnetic modes provide the "pairing glue" responsible for high-temperature superconductivity in the doped cuprates [1]. It is therefore extremely important to determine the nature of magnetism in these materials. Recent resonant inelastic x-ray scattering (RIXS) experiments in hole-doped cuprates [2-4] find a peak at energy transfers of $\sim 200-300 \mathrm{meV}$ that varies little in location and intensity between the undoped and heavily overdoped parts of the phase diagram. Previous work [2-4] has interpreted this peak as the signature of a collective magnetic mode that evolves with doping from a coherent magnon in the undoped antiferromagnet to a paramagnon with very little loss of spectral weight. This interpretation is troubling for several reasons. First, overdoped cuprates are good metals and well described by Fermi liquid theory [5]; there is little reason to believe that their propensity for magnetism is comparable to that of the undoped antiferromagnetic Mott insulator. Second, while inelastic neutron scattering experiments detect magnetic collective modes in the undoped cuprates, they find that the spectral weight for magnetic excitations above $100 \mathrm{meV}$ decreases dramatically with doping [6-10]. Finally, superconductivity in the cuprates vanishes rapidly with overdoping and it would be difficult to reconcile this with a magnetic pairing glue if the spectral weight of magnetic excitations did not simultaneously decrease.

Despite the wide range of doping in RIXS experiments, most theoretical work has focused on Mott insulating phases [11-13] in parent materials and local cluster models of doped materials $[12,14]$. Consequently, data from materials with itinerant electrons have been interpreted in terms of models of insulators. In particular, the hypothesis that RIXS data on optimally- and over-doped cuprates exhibit signs of collective magnetic modes is supported by analogy to spin wave theories that apply to undoped Mott-insulating cuprates and not by calculations on a microscopic model with mobile electrons. As even the underdoped cuprates exhibit a Fermi surface [15-17], this analogy is likely to fail over much of the cuprate phase diagram. This situation is especially unfortunate because it neglects processes in which one quasiparticle state is filled by photoabsorption and a different quasiparticle hole is left by reemission (Fig. 2), which constitutes the ubiquitous lowest-order contribution to RIXS in any itinerant electron system [18]. In this Letter we analyze RIXS in the doped cuprates with a more appropriate single-band quasiparticle model in order to decide whether one must invoke a higher-order contribution from collective modes to explain experimental data. We find that quasiparticles alone yield excellent quantitative agreement between theory and experiment. We also address the issue of whether our model is not only sufficient but necessary to explain experiments, and propose a simple measurement to conclusively distinguish quasiparticles from collective modes in RIXS.

Below, we calculate RIXS spectra using a model of noninteracting quasiparticles but including an interaction with a positively charged core hole via an exact determinantal method. We account for spin-orbit splitting of the core level in direct RIXS, in which a $\mathrm{Cu} 2 p$ core electron is photoexcited to the valence band, which opens a spin-flip (SF) channel in addition to the non-spin-flip (NSF) channel [19-21]. We apply our formalism to cuprates over a range of doping and achieve quantitative agreement with experimental data (Fig. 1). In particular, peaks in the calculated and measured line shapes disperse identically. As in experiments we also find that NSF line shapes are broader and higher in energy than SF line shapes. These features were previously attributed to magnetic effects, but we find that band structure alone produces dispersing line shapes, while the core hole combines with Pauli blocking to separate SF and NSF line shapes. 


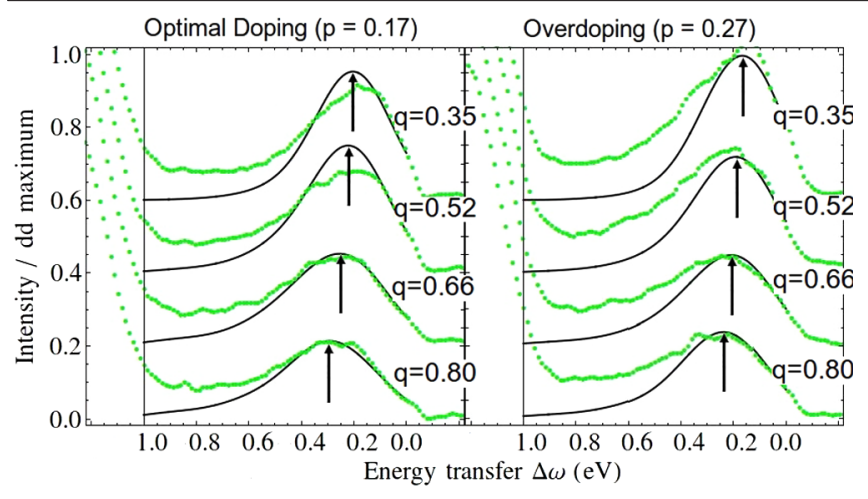

FIG. 1 (color online). Calculated (black curve) and experimental (Ref. [3], green dots) intensity vs energy transfer for spin-flip RIXS of optimally doped and overdoped Tl-2201 for antinodal momenta $\mathbf{Q}=q(\pi / a, 0)$ exhibiting identical dispersing peaks. We subtracted a nonresonant elastic peak from the raw experimental data. We did not remove the contribution of $d d$ excitations, which accounts for the discrepancy at large $\Delta \omega$.

Theoretical formalism.-Incident photons with momentum $\mathbf{q}$ and energy $\omega$ scatter into outgoing momentum $\mathbf{q}+\mathbf{Q}$ and energy $\omega-\Delta \omega$, where $\mathbf{Q}$ and $\Delta \omega$ are the momentum and energy transfer, with intensity $[18,20,21]$ $I \propto \sum_{f}\left|A_{f}\right|^{2} \delta\left(E_{f}-E_{i}-\Delta \omega\right)$, where $A_{f}$ is given by the Kramers-Heisenberg formula,

$$
A_{f}=\sum_{m} e^{i \mathbf{Q} \cdot \mathbf{R}_{m}} \chi_{\rho \sigma}\left\langle f\left|d_{m \rho}\left(H_{m}+\omega-E_{i}+i \Gamma\right)^{-1} d_{m \sigma}^{\dagger}\right| i\right\rangle
$$

Here $|i, f\rangle$ and $E_{i, f}$ are the initial and final electron states and energies, $d_{m \sigma}^{\dagger}$ creates a valence electron of spin $\sigma$ at site $m, \quad H_{m}=H+V_{m}$ is the sum of the equilibrium Hamiltonian $H$ and the potential $V_{m}$ due to a core hole at site $m$, and $\chi_{\rho \sigma}$ is a polarization-dependent $2 \times 2$ spin
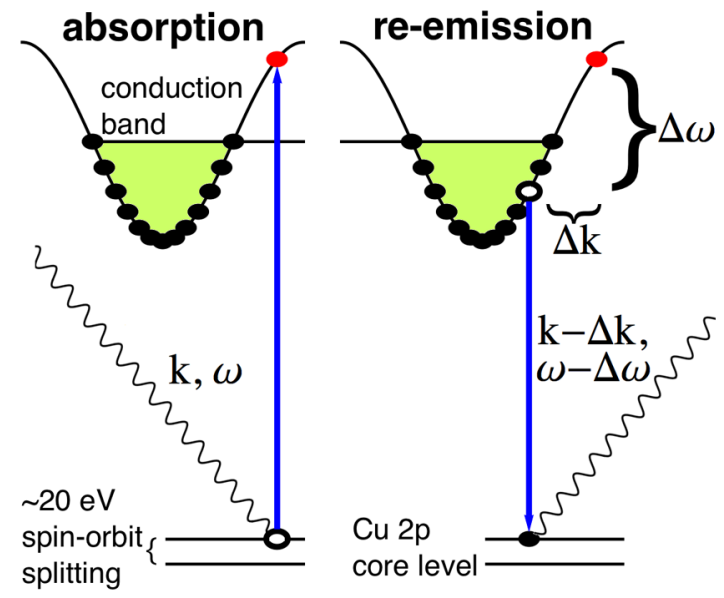

FIG. 2 (color online). Direct RIXS process that leaves behind a single particle-hole pair. The transient core hole potential can excite multiple particle-hole pairs. matrix that comes from the product of two dipole matrix elements: $\left\langle 3 d_{\sigma}\left|T_{\sigma}\right| 2 p\right\rangle$ and $\left\langle 2 p\left|T_{\rho}\right| 3 d_{\rho}\right\rangle$ for absorption and emission, where $T$ is a dipole transition operator [22]. In direct RIXS the strong $(\sim 20 \mathrm{eV})$ spin-orbit coupling of the $\mathrm{Cu} 2 p$ core level implies that the energy eigenstates $\left|2 p_{J, m}\right\rangle$ are not eigenstates of spin $S_{z}$. Thus off-diagonal elements $\rho \neq \sigma$ are permitted. One can isolate either the diagonal (NSF) or off-diagonal (SF) component of $\chi$ by varying only the incident polarization [22]. The immobile intermediate state core hole does not appear explicitly in Eq. (1) but affects the valence system indirectly. It forces absorption and emission to occur on the same site $m$ and contributes a width $\Gamma$ and the potential $V_{m}$ acting on valence electrons.

Because of the core hole, the eigenstates of $H_{m}$ have no simple relation to those of $H$ and it is convenient to work in the time domain. This puts the intensity in the form [23]

$$
\begin{aligned}
I \propto & \int_{-\infty}^{\infty} d s \int_{0}^{\infty} d t \int_{0}^{\infty} d \tau e^{i \omega(t-\tau)-i s \Delta \omega-\Gamma(t+\tau)} \\
& \times \sum_{m n} e^{i \mathbf{Q} \cdot\left(\mathbf{R}_{m}-\mathbf{R}_{n}\right)} \chi_{\rho \sigma} \chi_{\mu \nu} S_{\rho \sigma \mu \nu}^{m n}, \\
S_{\rho \sigma \mu \nu}^{m n}= & \left\langle e^{i H \tau} d_{n \rho} e^{-i H_{n} \tau} d_{n \sigma}^{\dagger} e^{i H s} \ldots d_{m \mu} e^{i H_{m} t} d_{m \nu}^{\dagger} e^{-i H(t+s)}\right\rangle .
\end{aligned}
$$

One obtains Eq. (2) via the identities $1 / z=\int_{0}^{\infty} e^{-z t} d t, \delta(z)=\int e^{i s z} d s$, replacing eigenvalues by operators, and recognizing resolutions of unity, $\quad$ e.g., $\quad \sum_{f}|f\rangle\langle f|\ldots| i\rangle \delta\left(E_{f}-E_{i}-\Delta \omega\right) \rightarrow$ $\int d s e^{-i \Delta \omega s} e^{i H s} \ldots e^{-i H_{i} s}|i\rangle$. However, Eq. (3) is best understood as a history of absorption and emission events separated by time evolution operators, that is, as the time-dependent amplitude to scatter a photon. The intensity is obtained from the square of this amplitude; hence, the pair of creation and annihilation operators is followed by its Hermitian conjugate.

In the following analysis we treat the valence band as a system of noninteracting quasiparticles. This approximation is valid when the quasiparticle lifetime is long compared to the core hole lifetime $1 / \Gamma$, in which case an electron is unlikely to scatter in the brief time between absorption and emission. In the cuprates, for example, typical values are $\Gamma=300-500 \mathrm{meV}$, which exceeds quasiparticle widths even quite far from the Fermi surface. (We stress that negligible quasiparticle scattering on short time scales is conceptually distinct from a Fermi liquid ground state [24]; the former, for example, implies nothing about de transport.)

If not for the insertion of $d$ and $d^{\dagger}$ operators, we could easily evaluate the many-body average in Eq. (3) in terms of the matrices $h_{(m, n)}$, where lowercase letters denote the matrix elements of a quadratic operator: $H=d_{i}^{\dagger} h_{i j} d_{j}$, and the Fermi occupancy matrix $N \equiv\left(1+e^{\beta h}\right)^{-1}$. A wellknown identity $[25,26]$ for the average of exponentiated quadratic operators $\left\langle e^{Z}\right\rangle=\operatorname{det}\left[(1-\hat{N})+e^{z} \hat{N}\right]$ extends 
naturally to the product of such exponentials via the BakerCampbell-Haussdorff lemma [27]. To compute $S_{\rho \sigma \mu \nu}^{m n}$ for direct RIXS we extend a method applied to tunneling in quantum wires [28] and resonant elastic x-ray scattering [29], which involved matrix elements like those in Eq. (3) but with one $d$ and one $d^{\dagger}$. We present the straightforward but lengthy derivation in the Supplemental Material [30]. The result is

$$
\begin{aligned}
S_{\rho \sigma \mu \nu}^{m n}= & \operatorname{det}(F)\left[\left\langle n \rho\left|(1-N) F^{-1} e^{-i h_{n} \tau}\right| n \sigma\right\rangle\right. \\
& \times\left\langle m \mu\left|e^{-i h s} e^{i h_{n} \tau}(1-N) F^{-1} U_{m n}\right| m \nu\right\rangle \\
& +\left\langle n \rho\left|(1-N) F^{-1} U_{m n}\right| m \nu\right\rangle \\
& \left.\times\left\langle m \mu\left|e^{i h_{m} t} U_{0} N F^{-1} e^{-i h_{n} \tau}\right| n \sigma\right\rangle\right],
\end{aligned}
$$

where $\quad U_{m n}=e^{-i h_{n} \tau} e^{i h s} e^{i h_{m} t}, \quad U_{0}=e^{i(\tau-t-s) h}, \quad$ and $F=1-N+U_{m n} U_{0} N$. For a full band $S$ vanishes, as it should, and for an empty band it reduces to $\left\langle n\left|e^{-i h_{n} \tau}\right| n\right\rangle\left\langle m\left|e^{i h_{m} t}\right| m\right\rangle$, a general term in the expression $\left|\sum_{m} \int G_{3 d}^{m m}(t) d t\right|^{2}$. That is, the amplitude of RIXS in an empty band is the coherent sum of electron propagators that start and end at the same core hole site. Equation (4) pertains to a full spin-orbital basis, but for a spin-independent Hamiltonian easily factorizes. If $H$ contains a singlet pairing term $d_{m, \uparrow}^{\dagger} B_{m n} d_{m, \downarrow}^{\dagger}$ it can be put into nonanomalous form suitable for matrix manipulations via a transformation $d_{m \uparrow}^{\dagger} \leftrightarrow d_{m \uparrow}$. This handles all spin density waves and pairing terms that occur in the cuprates. More complex spin density waves and triplet pairing require a more sophisticated formalism [31].

Results.-We now apply this formalism to study cuprate superconductors, comparing our results to experiments on $\mathrm{Tl}_{2} \mathrm{Ba}_{2} \mathrm{CuO}_{6+\delta}$ (Tl-2201) and $\mathrm{Bi}_{2} \mathrm{Sr}_{2} \mathrm{CuO}_{6+x}$ (Bi-2212). An outstanding puzzle is the existence of peaks in direct RIXS not seen in neutron scattering [7] or indirect RIXS [32]. We use the single-band model $H=\sum_{\mathbf{k}, \sigma} \varepsilon_{\mathbf{k}} d_{\mathbf{k}, \sigma}^{\dagger} d_{\mathbf{k}, \sigma}$, where $\varepsilon_{\mathbf{k}}=-2 t_{1}\left[\cos \left(k_{x}\right)+\right.$ $\left.\cos \left(k_{y}\right)\right]-4 t_{2} \cos \left(k_{x}\right) \cos \left(k_{y}\right)-2 t_{3}\left[\cos \left(2 k_{x}\right)+\cos \left(2 k_{y}\right)\right]-$ $4 t_{4}\left[\cos \left(2 k_{x}\right) \cos \left(k_{y}\right)+\cos \left(k_{x}\right) \cos \left(2 k_{y}\right)\right]$, using canonical tight-binding band structures fit to angle-resolved photoemission spectroscopy data: $\left(t_{1}, t_{2}, t_{3}, t_{4}\right)=$ $(126,-36,15,1.5) \mathrm{meV}$ for $\mathrm{Bi}-2212 \quad[33]$ and $\left(t_{1}, t_{2}, t_{3}, t_{4}\right)=(181,-75,-4,10) \mathrm{meV}$ for Tl-2201 [34]. We assume an attractive contact potential $V_{m}=$ $-U_{c} \sum_{\sigma} d_{m \sigma}^{\dagger} d_{m \sigma}$ for the core hole. We fix $\omega$ at the absorption maximum as in experiments. Figure 1 shows SF intensity versus $\Delta \omega$ for antinodal momenta $\mathbf{Q} \|(\pi, 0)$ in optimally doped and overdoped Tl-2201 ( $p=0.17$ and $p=0.27$ ) with $U_{c}=1.0 \mathrm{eV}$ along with data from Ref. [3]. We subtracted a Gaussian elastic peak at $\Delta \omega=0$ from all experimental data and convolved calculated line shapes with Gaussian functions of width equal to the instrumental resolutions of the corresponding experiments. We choose $U_{c}=1.0 \mathrm{eV}$ to obtain the best fit to NSF line shapes; SF RIXS is nearly independent of $U_{c}$. The most striking feature is an intensity peak that disperses to higher energy with increasing momentum, reaching a maximum of $250-300 \mathrm{meV}$, as seen in experiments [2-4,35,36]. One possible interpretation is that these peaks are due to inelastic scattering of a collective mode. However, we see that band structure alone can produce them [37]. Quantitatively, the calculated and experimental line shapes agree very well, with the location of peaks and their lowenergy side in nearly perfect agreement. There is a systematic discrepancy at large values of $\Delta \omega$ due to the tail of orbital $d d$ excitations [38]. It is reassuring that this discrepancy is nearly independent of momentum, as local excitations ought to be. The calculated nodal and antinodal line shapes also agree very well with experimental data from optimally doped Bi-2212 [39].

In Fig. 3 we show that the agreement between theory and experiment extends to NSF scattering. This is important because the SF and NSF channels correspond to spin and charge degrees of freedom and a difference in their line shapes is seen as compelling proof of magnetic physics. Indeed, the matrix elements in Eq. (5), below, are manifestly spin independent, so that SF and NSF line shapes should be identical in the absence of interactions. However, the core hole potential dramatically separates SF and NSF line shapes. As $U_{c}$ increases, the NSF peak moves to higher energies and broadens while the SF peak remains relatively sharp, exactly as seen in experiments. For $U_{c} \sim 1.0 \mathrm{eV}$ the agreement is very good up to energies at which the $d d$ tail becomes significant.

The core hole separates SF and NSF line shapes as follows: Its attractive potential tends to keep the photoexcited electron of spin $\sigma$ bound near $\mathbf{R}_{m}$, leading to elastic scattering. Pauli blocking prevents other electrons of spin $\sigma$ from hopping onto $\mathbf{R}_{m}$ and filling the core hole, thereby robbing spectral weight from inelastic scattering. With

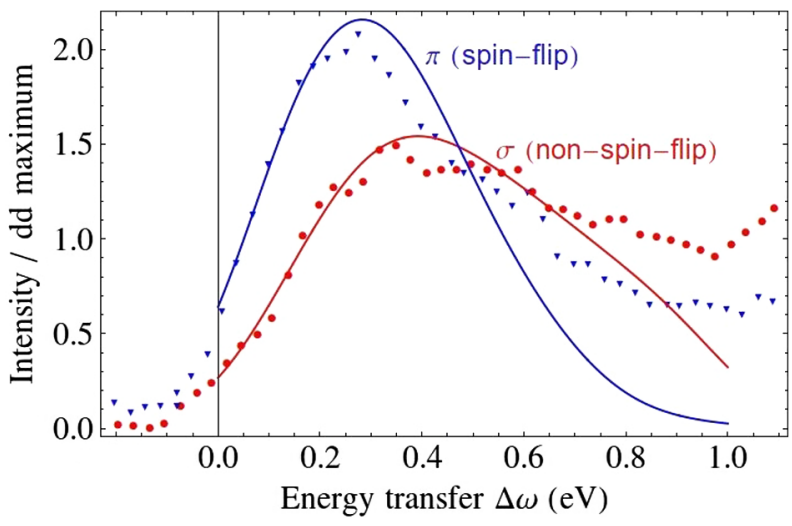

FIG. 3 (color online). Spin-flip ( $\pi$-polarized) and non-spin-flip ( $\sigma$-polarized) antinodal $\mathbf{Q}=0.80(\pi / a, 0)$ line shapes of optimally doped $(p=0.15) \mathrm{Bi}-2212$ for core hole potential $U_{c}=1.0 \mathrm{eV}$. Blue: spin-flip channel $/ \pi$ polarization; red: nonspin-flip channel $/ \sigma$ polarization. Solid lines: calculated results; triangles, circles: $\pi$ - and $\sigma$-polarized data from Ref. [35]. 
sufficient energy the photoexcited electron may be dislodged, allowing inelastic scattering. Hence NSF scattering with small $\Delta \omega$ is suppressed relative to scattering with large $\Delta \omega$. This argument does not apply to SF scattering because spin- $\bar{\sigma}$ electrons are not Pauli blocked. This explains the observed difference in SF versus NSF line shapes as well as the insensitivity of NSF line shapes to the core hole. Because this effect is nonperturbative, an exact analysis is indispensable for detecting it. Since NSF line shapes can be calculated reasonably well without a core hole potential a formula for the RIXS intensity in this limit may be useful. We obtain

$$
\begin{aligned}
I \propto & \sum_{\alpha, \beta}\left|\frac{\sum_{\mathbf{k}} \chi_{\rho \sigma}\langle\alpha \mid \mathbf{k}+\mathbf{Q}, \rho\rangle\langle\mathbf{k}, \sigma \mid \beta\rangle}{\omega-\varepsilon_{\beta}+i \Gamma}\right|^{2} \\
& \times n_{f}\left(\varepsilon_{\alpha}\right)\left[1-n_{f}\left(\varepsilon_{\beta}\right)\right] \delta\left(\varepsilon_{\alpha}-\varepsilon_{\beta}-\Delta \omega\right),
\end{aligned}
$$

where $|\mathbf{k}, \mathbf{k}+\mathbf{Q}\rangle$ are momentum eigenstates and $|\alpha, \beta\rangle$ are single-particle eigenstates of $H$ in the spin-orbital basis.

Finally, we note that the overall intensity of calculated and measured line shapes changes very little from the optimally doped to the overdoped material. Our calculations differ from measured data by some overall multiplicative constant due to self-absorption, properties of the apparatus, and other factors. However, we used the same constant for all line shapes in Fig. 1, and thus the nearly identical intensities at different dopings in our calculations are meaningful. If the RIXS signal came predominantly from a collective mode this would imply a spectral weight that varies little with doping. In our model, however, this happens naturally because a slight change in chemical potential does not strongly affect the band structure.

Summary and outlook.-We derived a formalism to treat band structures, pairing, and core hole potentials in direct and indirect RIXS. The line shapes we calculated in a single-band model of mobile electrons agreed with experiments over a wide range of doping for both spin-flip and non-spin-flip scattering, and we found a mechanism by which the core hole differentiates the two channels. We concluded that dispersing peaks seen in RIXS experiments on cuprates can be attributed to band structure alone without invoking collective modes. Thus the constant intensity of peaks in RIXS as doping increases does not imply a constant spectral weight of magnetic excitations, which has important implications for the mechanism of superconductivity in these materials [1].

Our model of noninteracting quasiparticles is a priori well supported by experimental evidence for the overdoped cuprates [5]. The agreement of our model with measured data suggests that it remains valid to doping at least as low as $p=0.15$. We expect that it would work as far as $p=0.08$, where a Fermi surface is found in experiments [15-17]. However, a noninteracting model becomes insufficient at some point in the underdoped regime. The analysis

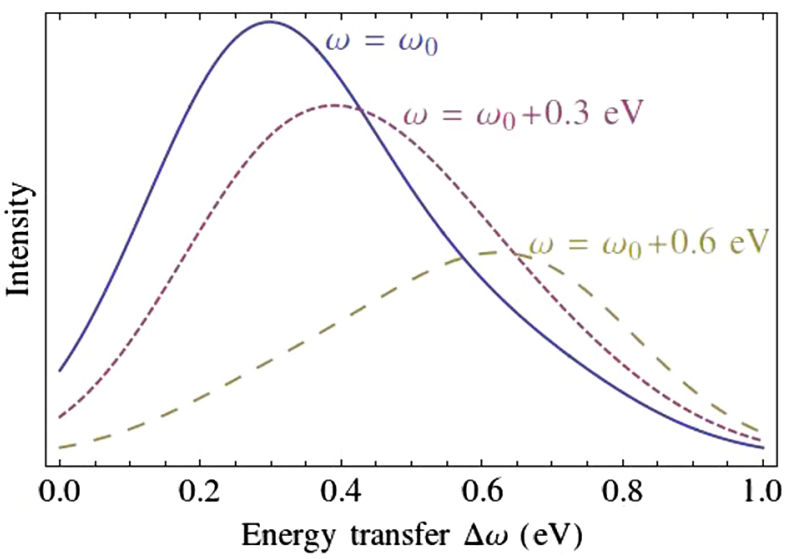

FIG. 4 (color online). Calculated intensity vs energy transfer for spin-flip RIXS of optimally doped Bi-2212 $(p=0.15)$ for antinodal momentum $\mathbf{Q}=(\pi / a, 0)$ at incident energies $0,0.2$, and $0.4 \mathrm{eV}$ above the absorption maximum. The increase in $\Delta \omega$ with $\omega$ occurs when the RIXS final state belongs to the particlehole continuum and does not occur if the final state is an excitation of a collective mode.

presented in this Letter can be extended to deeply underdoped antiferromagnetic states via a RPA-like analysis, which is known to correctly reproduce spin wave excitations in the insulating state. As the insensitivity of RIXS line shapes to doping in the range we have considered persists to some extent to the undoped Mott antiferromagnet, a theory that bridges these two limits is very desirable.

While we showed above that a model of noninteracting quasiparticles is in excellent agreement with RIXS experiments, we have not yet presented a prediction that contrasts interpretations in terms of quasiparticles and in terms of collective modes. Such evidence, however, could easily be obtained by measuring RIXS line shapes for incident energy $\omega$ above the absorption maximum. As shown in Fig. 4, as $\omega$ increases line shapes move to larger $\Delta \omega$. The RIXS signal due to inelastic scattering of a collective mode does not behave this way because $\Delta \omega$ cannot exceed the energy of the mode. (This simple qualitative test could also prove useful in the analysis of RIXS in the Fe-based superconductors [40]. It would be interesting to apply our formalism to such systems, where an additional complicating factor is the existence of multiple bands.) This exemplifies that one must calculate the RIXS signal itself, and not a proxy such as magnetic susceptibility. A susceptibility $\chi(\omega, \mathbf{k})$ depends on a single frequency $\omega$, which corresponds to the energy of excitations. Since the energy transfer in RIXS plays a similar role, the correspondence $\Delta \omega$ (RIXS) $\rightarrow \omega$ (susceptibility) is often assumed. However, this neglects the significant interplay of $\omega$ and $\Delta \omega$ in RIXS. In RIXS the phase space for final states is modified by the intermediate state resonance. For example, in Fig. 4 the intermediate state photoelectron's energy increases with $\omega$, which tends to increase the energy of the final state particle-hole pair. 
We acknowledge Peter Abbamonte for spurring our interest in RIXS, Mark Dean and Mathieu LeTacon for sharing data and helpful feedback, and Dmitry Abanin, Jeroen van den Brink, Marco Grioni, and Henrik Ronnow for discussions. We acknowledge support from HarvardMIT CUA, the ARO MURI on Atomtronics, and the ARO MURI Quism program.

[1] D. J. Scalapino, Rev. Mod. Phys. 84, 1383 (2012).

[2] M. Le Tacon et al., Nat. Phys. 7, 725 (2011).

[3] M. Le Tacon et al., Phys. Rev. B 88, 020501 (2013).

[4] M. Dean and G. Dellea, Nat. Mater. 12, 1019 (2013).

[5] M. R. Norman et al., Nature (London) 392, 157 (1998).

[6] R. J. Birgeneau, C. Stock, J. M. Tranquada, and K. Yamada, J. Phys. Soc. Jpn. 75, 111003 (2006).

[7] S. Wakimoto, K. Yamada, J. M. Tranquada, C. D. Frost, R. J. Birgeneau, and H. Zhang, Phys. Rev. Lett. 98, 247003 (2007).

[8] C. Stock, R. A. Cowley, W. J. L. Buyers, C. D. Frost, J. W. Taylor, D. Peets, R. Liang, D. Bonn, and W. N. Hardy, Phys. Rev. B 82, 174505 (2010).

[9] J. M. Tranquada, G. Xu, and I. A. Zaliznyak, J. Magn. Magn. Mater. 350, 148 (2014).

[10] See, however, Refs. [36] and [35], which argue that the absence of magnetic signatures in neutron scattering is not relevant.

[11] J. van den Brink, Europhys. Lett. 80, 47003 (2007).

[12] F. Vernay, B. Moritz, I. S. Elfimov, J. Geck, D. Hawthorn, T. P. Devereaux, and G. A. Sawatzky, Phys. Rev. B 77, 104519 (2008).

[13] C.-C. Chen et al., Phys. Rev. Lett. 105, 177401 (2010).

[14] C. J. Jia, E. A. Nowadnick, K. Wohlfeld, C. C. Chen, S. Johnston, T. Tohyama, B. Moritz, and T. P. Devereaux, Nat. Commun. 5, 3314 (2014).

[15] D. LeBoeuf et al., Nature (London) 450, 533 (2007).

[16] N. Doiron-Leyraud, C. Proust, D. LeBoeuf, J. Levallois, J.-B. Bonnemaison, R. Liang, D. A. Bonn, W. N. Hardy, and L. Taillefer, Nature (London) 447, 565 (2007).

[17] B. Vignolle et al., C.R. Phys. 12, 446 (2011).

[18] A. Kotani and S. Shin, Rev. Mod. Phys. 73, 203 (2001).
[19] L. J. P. Ament, G. Ghiringhelli, M. M. Sala, L. Braicovich, and J. van den Brink, Phys. Rev. Lett. 103, 117003 (2009).

[20] L. Ament, M. van Veenendaal, T. Devereaux, J. Hill, and J. van den Brink, Rev. Mod. Phys. 83, 705 (2011).

[21] M. W. Haverkort, Phys. Rev. Lett. 105, 167404 (2010).

[22] We explicitly derive the effects of spin-orbit coupling in the Supplemental Material [30]. See also Refs. [20] and [21]

[23] P. Nozières and E. Abrahams, Phys. Rev. B 10, 3099 (1974).

[24] X. Deng, J. Mravlje, R. Žitko, M. Ferrero, G. Kotliar, and A. Georges, Phys. Rev. Lett. 110, 086401 (2013).

[25] D. J. Scalapino and R. L. Sugar, Phys. Rev. Lett. 46, 519 (1981).

[26] R. T. Scalettar, D. J. Scalapino, and R. L. Sugar, Phys. Rev. B 34, 7911 (1986).

[27] I. Klich, in Quantum Noise in Mesoscopic Physics, edited by Y. Nazarov (Springer, New York, 2003).

[28] D. A. Abanin and L. S. Levitov, Phys. Rev. Lett. 94, 186803 (2005).

[29] D. Benjamin, D. Abanin, P. Abbamonte, and E. Demler, Phys. Rev. Lett. 110, 137002 (2013).

[30] See Supplemental Material at http://link.aps.org/supplemental/ 10.1103/PhysRevLett.112.247002 for explicit calculation of spin-orbit effects and a derivation of Eq. (4).

[31] I. Klich, arXiv:1403.7824.

[32] J. Hill, G. Blumberg et al., Phys. Rev. Lett. 100, 097001 (2008).

[33] R. S. Markiewicz, S. Sahrakorpi, M. Lindroos, H. Lin, and A. Bansil, Phys. Rev. B 72, 054519 (2005).

[34] D. C. Peets, J. D. F. Mottershead, B. Wu, I. S. Elfimov, R. Liang, W. N. Hardy, D. A. Bonn, M. Raudsepp, N. J. C. Ingle, and A. Damascelli, New J. Phys. 9, 28 (2007).

[35] M. P. M. Dean et al., Phys. Rev. Lett. 110, 147001 (2013).

[36] M. P. M. Dean et al., Nat. Mater. 11, 850 (2012).

[37] Zeyher and Greco make a similar claim about Raman spectra in Ref. [41] and argue that RIXS behaves similarly.

[38] J. Zaanen and G. Sawatzky, J. Solid State Chem. 88, 8 (1990).

[39] H. Ronnow (unpublished).

[40] K.-J. Zhou et al., Nat. Commun. 4, 1470 (2013).

[41] R. Zeyher and A. Greco, Phys. Rev. B 87, 224511 (2013). 